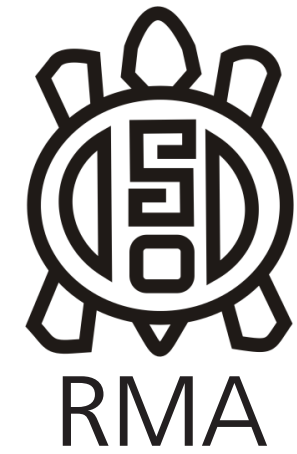

Arqueología

\section{La fauna de Loma l'Ántigo. Primeros datos zooarqueológicos del Período Desarrollos Regionales del valle del Cajón, Catamarca, ca. 1200-1500 d.C.}

\author{
The fauna from Loma l'Ántigo. First zooarchaeological data \\ on the Regional Developments Period from the Cajón valley, \\ Catamarca, ca. 1200-1500 AD
}

Carlos Raúl Belotti López de Medina* y María Fabiana Bugliani**

\begin{abstract}
Resumen
Se reportan los resultados del análisis de las arqueofaunas del sitio Loma l'Ántigo del valle del Cajón (Catamarca). Loma l'Ántigo es un sitio del Período Desarrollos Regionales con un patrón de asentamiento aglomerado y emplazado sobre la cima aplanada de una loma. La fauna analizada proviene de nueve sondeos y la excavación en área de tres recintos (Estructuras E25, E93 y E66). Camelidae es la familia más frecuente en el sitio y los conjuntos, registrándose especímenes óseos comparables a especies domésticas y silvestres y perfiles de supervivencia/mortandad que incluyen animales juveniles, subadultos y adultos/viejos. Los conjuntos de Loma l'Ántigo no muestran diferencias significativas respecto de sitios formativos del valle del Cajón (Yutopián y Cardonal), lo que indica una continuidad global de los modos de explotación de fauna y de los condicionantes naturales y culturales de las historias tafonómicas, pese a los cambios socioculturales, políticos y ambientales postulados entre el primer y segundo milenio de la Era para el sur de los valles Calchaquíes.
\end{abstract}

Palabras clave: Valle del Cajón; Zooarqueología; Desarrollos Regionales.

\begin{abstract}
This paper reports the results of the analyses conducted on the archaeofaunas from the Loma l'Ántigo archaeological site (Cajón valley, Catamarca province). Loma l'Ántigo is a conglomerate settlement that rests on the top of a flattened hill, and which was dated to the Regional Developments archaeological period. The archaeofaunas were recovered from nine test-pits and the area excavation of three enclosures (structures E25, E66 and E93). Camelidae is both the most ubiquitous family between assemblages and the most abundant across them. Camelid specimens comparable to both domestic and wild species were recorded, and age profiles include young, subadult, adult and old animals. Loma l'Ántigo assemblages do not exhibit significant differences against archaeofaunas dated to the Formative Period of the Cajón valley (Cardonal and Yutopián sites), pointing to a broad continuity of modes of faunal exploitation and to similar environmental and sociocultural constraints on taphonomic histories, despite previously posited demographic, political and climate changes around the beginning of the second millennium AD for the Southern Calchaqui valleys.
\end{abstract}

Keywords: Cajón valley; Zooarchaeology; Regional Developments.

\section{Introducción}

La zooarqueología de la etapa agroalfarera del sur de los valles Calchaquíes (Provincias de Catamarca y Tucumán), área que comprende los faldeos occidentales de las Sierras del Aconquija y los valles de Yocavil y del Cajón, tuvo un importante desarrollo en los últimos 20 años (e. g. Belotti
López de Medina, 2011, 2015a, 2016c, 2016b; Izeta, 2007b, 2007a; Izeta \& Cortés, 2006; Nastri et al., 2012; Pratolongo, 2008). A esto se suman los antecedentes de la Quebrada de Tolombón y del valle Calchaquí en el sector norte de la misma cuenca (Provincia de Salta) ( $e$. g. Belotti López de Medina, 2015b; de Guerrero, 1968; Izeta et al., 2009; Mengoni Goñalons, 1991, 2013). 
Los antecedentes abarcan los períodos Formativo (ca. 100 a.C. - 900 d.C.), Desarrollos Regionales o Tardío (ca. 900 - 1450 d.C.) e Inka (ca. 1450 - 1536 d.C.). Camelidae es la familia dominante a lo largo de la secuencia y se identificaron especímenes comparables a especies silvestres (guanaco, vicuña) y domésticas (llama). Los sitios se ajustan por lo tanto a lo propuesto para otras regiones del Noroeste argentino: establecimiento de una estrategia mixta de ganadería y caza de camélidos (Escola, 2002; Olivera, 1997; Yacobaccio et al., 1997).

El registro zooarqueológico insinúa algunos cambios durante los últimos dos períodos (Desarrollos Regionales e Inka, desde ca. 900 d.C), en coincidencia con transformaciones demográficas, económicas y políticas de las sociedades calchaquíes meridionales (ver González \& Tarragó, 2005; Tarragó, 2000). Desde fines del Formativo (ca. el 1100 rcbp) se observa una mayor frecuencia de fauna menor en los conjuntos (roedores, dasipódidos, aves, etc.) y un incremento simultáneo de la frecuencia de especímenes osteológicamente maduros de Camelidae (Belotti López de Medina, 2015a; Izeta, 2007b). También se documentó una mayor frecuencia de especímenes en el rango de tamaño de llama en sitios tardíos del Valle de Yocavil (Belotti López de Medina, 2015a; Pratolongo, 2008). Con la excepción de Molinos 1 (Izeta et al, 2009), el registro tardío del norte de los valles Calchaquíes sigue estas tendencias (Mengoni Goñalons, 1991, 2013; Belotti López de Medina 2015b).

Para el área de estudio de este reporte, el valle del Cajón (Catamarca), se cuenta con los análisis de los conjuntos de Yutopián y Cardonal (Izeta, 2007b, 2007a). Las dataciones de Yutopián se extienden entre 1940 y 1630 años rcbp (Izeta, 2007b; Scattolin \& Gero, 1997), mientras que Cardonal tiene una datación de $1878 \pm 57$ rcbp (Scattolin et al., 2007), ubicándose en los primeros siglos del Período Formativo de la región (Scattolin, 2006). Ambos sitios muestran el predominio de Artiodactyla y Camelidae y se identificaron especímenes de llama, vicuña y guanaco. No existen antecedentes zooarqueológicos del segundo milenio d.C. para el valle del Cajón; los conjuntos descritos en este reporte son los primeros del período Desarrollos Regionales y permitirán una aproximación preliminar a los cambios y continuidades del registro zooarqueológico en el área de estudio.

\section{Materiales y métodos}

\section{Proveniencia de la fauna, el sitio Loma l'Ántigo}

El valle del Cajón es una depresión longitudinal semiárida limitada al este por las sierras del Cajón y al oeste por las cumbres que se extienden desde el borde de Puna ( $e$. g. Las cuevas, Chango Real, Laguna Blanca). A lo largo del fondo de valle corre el Río Colorado-Santa María en dirección NS, el cual vira hacia el este al sur de la Sierra del Cajón y sube en dirección norte por el valle de Yocavil o Santa María (Turner, 1973). Loma l'Ántigo está emplazado sobre la cima aplanada de una loma de 60 metros de altura (2700 m snm) y laderas abruptas al norte de una planicie denominada Pampa Grande por Weiser (1922) (Figura 1). El poblado fue bautizado antes como Loma del pueblito á Guasamayo por Ten Kate (1893) y Pueblo Viejo de Pampa Grande por Weiser (1922). Weiser realizó el primer levantamiento planimétrico del sitio.

Los trabajos actuales en Loma l'Ántigo iniciaron en el año 2009 bajo la dirección de Bugliani $(2012,2018)$. En una primera etapa se condujo un nuevo levantamiento con brújula taquimétrica y se registraron las características arquitectónicas del sitio. Posteriormente se realizó una nueva planimetría con estación total (Figura 2), registrándose 111 recintos (Bugliani, 2018). En una primera aproximación se excavaron nueve sondeos en la cima, siete de ellos al interior de distintos recintos y dos por fuera de las habitaciones, además de una trinchera al

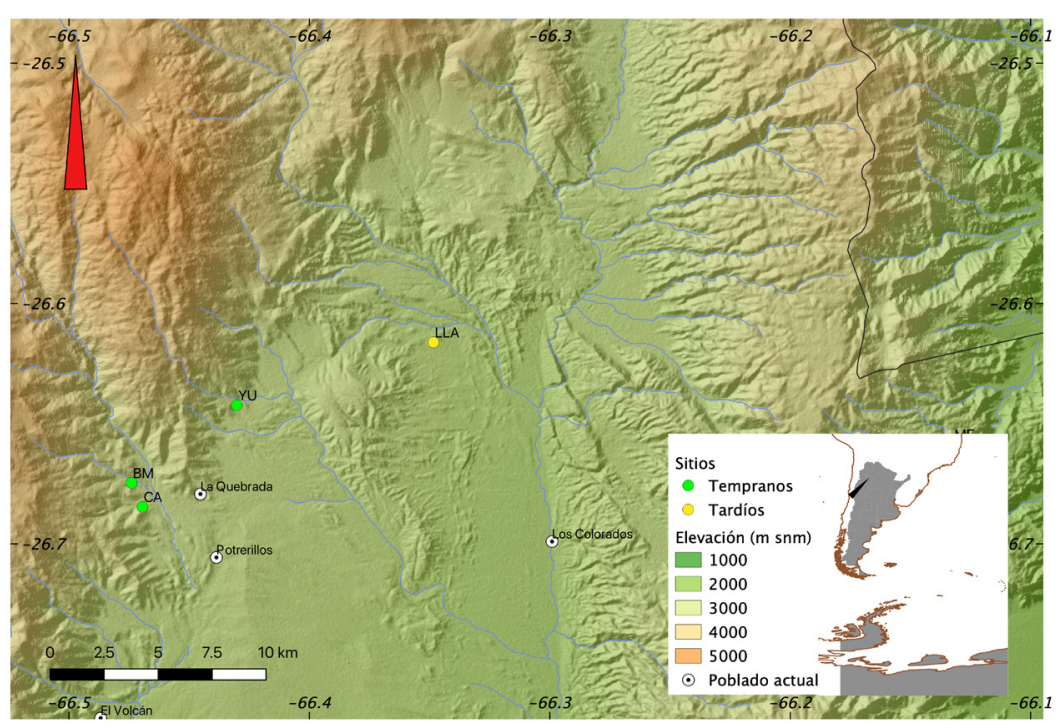

Figura 1. Sitios arqueológicos del valle del Cajón (sur de los valles Calchaquíes, Provincia de Catamarca). BM: Bordo Marcial. CA: Cardonal. LLA: Loma I'Ántigo. YU: Yutopian. Cartografía generada en QGis 3.10 en base a modelos de elevación digitales y vectores provistos por el Instituto Geográfico Nacional de la República Argentina.

Figure 1. Archaeological sites from the Cajon valley (southern Calchaqui valleys, Catamarca province). BM: Bordo Marcial. CA: Cardonal. LLA: Loma l'Ántigo. YU: Yutopian. Cartography generated under QGis 3.1 from digital elevation models and vectorial layers provided by the National Geographic Institute (Argentina). 


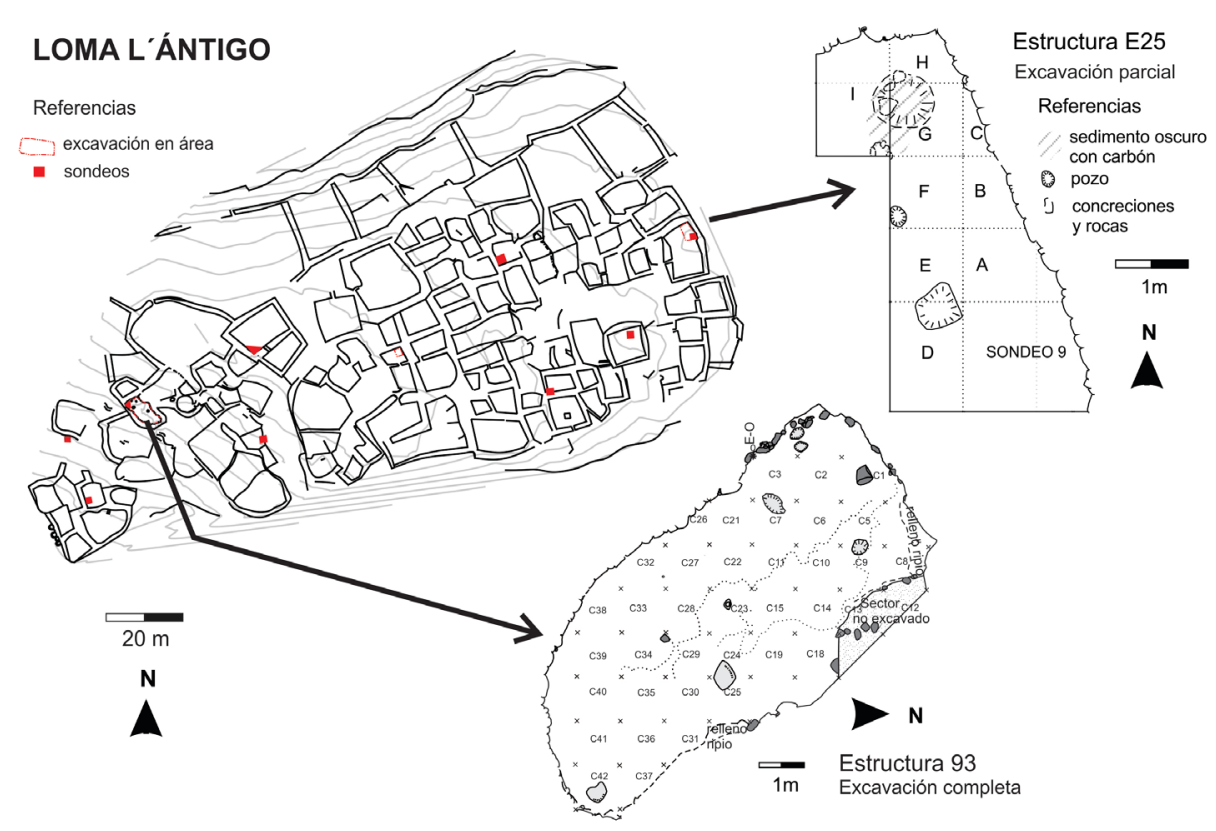

Figura 2. Loma l'Ántigo: Izquierda, plano del asentamiento indicando la localización de las estructuras y los sondeos y excavaciones; derecha arriba, plano de E25 y excavación; derecha abajo, plano de E93 y excavación.

Figure 2. Loma l'Ántigo. Left, plan of the settlement showing locations of structures, test-pits and area excavations; upper right, plan of structure 25 and excavation; lower right, plan of structure 93 and excavation.

pie de la loma. Nueve dataciones radiocarbónicas (Tabla 1) permitieron establecer el lapso de ocupación de Loma I'Ántigo entre fines del siglo XIII y fines del XV de la Era -fechas calibradas con un sigma- (Bugliani, 2018).

Posteriormente se ampliaron las excavaciones de las estructuras E66, E25 y E93. En las dos últimas se observaron pisos de ocupación bien definidos y con rasgos diferenciables. El piso de ocupación de E93 comienza a $25 \mathrm{~cm}$ de profundidad. Este presenta dos estructuras de combustión en cubeta, posibles hoyos de poste y otros pozos cavados en la roca base. Los artefactos líticos y cerámicos se encuentran dispersos en la extensión del piso, con una mayor concentración junto al muro sur del recinto, alrededor de las estructuras de combustión. La cerámica está dominada por fragmentos de los tipos Ordinario y Santa María Bicolor. Con excepción de una punta de proyectil de obsidiana y cuatro nódulos, el conjunto lítico se compone de lascas y desechos de andesita, cuarzo, riolita y obsidiana (Moro, 2016). La excavación de E25 reveló una estructura de combustión en cubeta y hoyos cavados en la roca base; los tipos cerámicos y las formas de las vasijas presentes son los mismos que en E93.

La fauna analizada proviene de los sondeos de las estructuras E8, E25, E39, E44, E93, E99 y E103, el sondeo

en un área entre estructuras -A16 y E82- y un sondeo al pie de la loma, y de las excavaciones en área de E25, E66 y E93. En total se recuperaron 714 especímenes óseos (sensu Salemme et al., 1988), que fueron agrupados en conjuntos por su asociación a los recintos y, por tratarse de conjuntos más abundantes y diversos provenientes de excavaciones en área, por criterios estratigráficos en el caso de los materiales de E25 y E93.

\section{Técnicas de análisis}

La caracterización biológica y tafonómica de los especímenes se llevó adelante en el Instituto de las Culturas (IDECU, Universidad de Buenos Aires, CONICET). Para la caracterización biológica se aplicó un abordaje morfológico y morfométrico (Hillson, 2005; Izeta et al., 2012; Menegaz et al., 1988; Pacheco Torres et al., 1979). Los especímenes fueron clasificados además en cinco categorías de tamaño corporal (Izeta, 2007b): 1. vertebrados muy pequeños (e. g. Ctenomys sp. 0,5 kg); 2 . pequeños (e. g. Lagidium viscacia 1,60 kg). 3. medianos (e. g. Pterocnemia pennata $30 \mathrm{~kg}$ ); 4. grandes (e. g. Hippocamelus antisensis $70 \mathrm{~kg}$ y Lama glama $120 \mathrm{~kg}$ );

Tabla 1. Fechados radiocarbónicos del sitio Loma I'Ántigo (fuente: Bugliani 2018, tabla 2).

Table 1. Radiocarbon dates from Loma l'Ántigo (source: Bugliani 2018, table 2).

\begin{tabular}{|c|c|c|c|c|c|c|}
\hline Código & Código laboratorio & Fechado & Calibrado $1 \sigma$ & Calibrado $2 \sigma$ & Procedencia & Material \\
\hline C842-8 & AA89386 & $680 \pm 33 \mathrm{AP}$ & $1278-1383$ & $1269-1390$ & Loma l'Ántigo E44, nivel 4 & Carbón nivel de ocupacion \\
\hline C831-7 & AA89385 & $663 \pm 34 \mathrm{AP}$ & $1283-1385$ & $1275-1394$ & Loma l'Ántigo Al6, nivel 2 & Carbón nivel de ocupacion \\
\hline C830-4 & AA89382 & $612 \pm 33 \mathrm{AP}$ & 1300-1395 & 1294-1404 & Loma l'Ántigo E99, nivel 4 & Carbón nivel de ocupacion \\
\hline C841-6 & AA89384 & $602 \pm 34 \mathrm{AP}$ & 1306-1398 & $1296-1408$ & Loma l'Ántigo E93, rasgo 1 & Carbón fogón \\
\hline C853-2 & AA89380 & $556 \pm 33 \mathrm{AP}$ & $1323-1418$ & $1307-1432$ & Loma l'Ántigo E25, nivel 2 & Carbón nivel de ocupacion \\
\hline C820-3 & AA893881 & $546 \pm 33 \mathrm{AP}$ & 1326-1424 & $1312-1436$ & Loma l'Ántigo E103, nivel 4 & Carbón nivel de ocupacion \\
\hline C1110-T4 & AA97849 & $516 \pm 34 \mathrm{AP}$ & $1422-1448$ & $1404-1458$ & Loma l'Ántigo E93, nivel 3 & Carbón área de combustión \\
\hline C833-5 & AA89383 & $471 \pm 44 \mathrm{AP}$ & $1326-1424$ & $1312-1436$ & Loma l'Ántigo E93, nivel 3 & Carbón fogón \\
\hline C802-1 & AA899379 & $432 \pm 35 \mathrm{AP}$ & $1431-1474$ & [1417-1515] [1598-1617] & Loma l'Ántigo E8, nivel 2 & Carbón nivel de ocupacion \\
\hline
\end{tabular}


9. tamaño indeterminado.

Para determinar los rangos etarios de los especímenes de Camelidae se utilizaron la epifización (Burger \& Salazar, 2003; De Nigris, 2004; Kent, 1982) y series de erupción y desgaste dentarios (Wheeler, 1982). Para la discusión de los perfiles obtenidos utilizamos las siguientes clases de edad como referencia: cría (0-12 meses), juvenil (1224 meses), subadulto (24-36 meses), adulto (3-7 años) y viejo (> 7 años) (Kaufmann et al., 2017).

Para la caracterización tafonómica se siguieron los criterios diagnósticos propuestos o compilados por distintos autores, registrándose alteraciones artificiales y naturales de la superficie de los huesos, morfología de fracturas, termoalteración, etc. (Behrensmeyer, 1978; Bunn, 1981; Fernandez-Jalvo \& Andrews, 2016; Fisher, 1995; Johnson, 1985; Lyman, 1994; Mengoni Goñalons, 1999; Morlan, 1983; Potts \& Shipman, 1981).

Los datos primarios se cargaron en una base de datos relacional bajo el sistema PostgreSQL 11 y se cuantificaron por medio de consultas SQL. Se utilizaron las siguientes medidas de abundancia: 1) número de especímenes (NSP, comprende elementos identificados y no identificados); 2) número de especímenes identificados (NISP). A nivel sitio utilizamos la ubicuidad taxonómica (número de contextos en los que está presente un taxón). Si bien todos los especímenes son identificables a algún nivel taxonómico (clase o phylum al menos), a efectos de la cuantificación consideramos como identificadas sólo a las caracterizaciones al rango de orden o inferior. Estas medidas serán interpretadas como de escala ordinal (Lyman, 2008; Wolverton et al., 2014). A fin de comparar con otros conjuntos del valle del Cajón, se procedió a la construcción de arqueotafogramas (Lyman, 1994; Mengoni Goñalons, 1999). Los análisis estadísticos y gráficas de datos se hicieron bajo el lenguaje R 4.0 (Carlson, 2017).

\section{Resultados}

La fauna recolectada a la fecha se compone de 714 especímenes, de los cuales se identificaron 222 al rango de orden o inferior (Tabla 2). El subconjunto de especímenes identificados está dominado a nivel orden por Artiodactyla (NISP 138, ubicuidad 6 incluyendo camélidos y cérvidos) y Rodentia (NISP 80, ubicuidad 3); el predominio corresponde a Camelidae a nivel familia (NISP 83, ubicuidad 5). Entre los restos no identificados predominan los de tamaño corporal indeterminado (tamaño 9, NSP 363) y los que pudieron asignarse a vertebrados medianos a grandes (tamaño 3 a 4, NSP 107). Estos patrones son consistentes con el registro agroalfarero del Noroeste, dominado por restos de camélidos y artiodáctilos indeterminados. Con frecuencia, se presume de los últimos que la mayoría pertenece a Camelidae, ya que los restos de cérvidos son muy infrecuentes (e. g. Izeta, 2007b; Rodriguez Loredo, 1992; Pratolongo, 2008).

La descripción mas detallada de los conjuntos se limitará a los contextos excavados en área y que brindaron el mayor volumen de evidencia: los pisos de las estructuras 25 y 93 (Tabla 2, NSP 575). En ambos casos la distribución horizontal de los especímenes es irregular, pudiendo tratarse de descartes primarios de facto sesgados por los patrones de uso y mantenimiento del espacio intramuros. Haciendo foco en los especímenes de vertebrados medianos a grandes (Figura 3), los que con más probabilidad son de origen antrópico, se observa su concentración al noroeste de la excavación de E25, espacio correspondiente a una acumulación de cenizas,

Tabla 2. Número de especímenes (NSP) por taxón, clase de tamaño y procedencia.

Table 2. Number of specimens (NSP) tallied by taxon, bodysize, and provenience.

\begin{tabular}{|c|c|c|c|c|c|c|c|c|c|c|c|c|}
\hline \multirow[b]{2}{*}{ Taxón } & \multirow[b]{2}{*}{ Tamaño } & \multicolumn{11}{|c|}{ NSP } \\
\hline & & E8 & E44 & E66 & E99 & E103 & Al6 & Pie de loma & E25 no piso & E25 piso & E93 no piso & E93 piso \\
\hline VERTEBRATA & 1 & & 1 & & & & & & & 1 & & 1 \\
\hline VERTEBRATA & $1-2$ & & & & & & & & & & 1 & \\
\hline VERTEBRATA & 2 & & & & & & 1 & & & 1 & & 1 \\
\hline VERTEBRATA & $2-3$ & & & & & & 1 & & & & 2 & 6 \\
\hline VERTEBRATA & 3 & & & & & & 2 & & & & & 3 \\
\hline VERTEBRATA & $3-4$ & 3 & 1 & & 3 & 5 & 3 & & 7 & 8 & 14 & 33 \\
\hline VERTEBRATA & 4 & & & & 1 & & 1 & & 1 & 3 & 8 & 11 \\
\hline VERTEBRATA & 9 & & 1 & 1 & 12 & 8 & 82 & 1 & 36 & 62 & 53 & 107 \\
\hline Artiodactyla & 3 & & & & & & & & 1 & & 8 & \\
\hline Artiodactyla & $3-4$ & & & & & & 3 & & & 3 & 2 & 3 \\
\hline Artiodactyla & 4 & 1 & & & 1 & & & & 3 & 16 & 1 & 12 \\
\hline Hippocamelus antisensis & 4 & & & & & 1 & & & & & & \\
\hline Camelidae & 3 & & & & 1 & 2 & & 2 & & & & 1 \\
\hline Camelidae & 4 & & & & & & & & 5 & 15 & 9 & 41 \\
\hline Lamasp. & 3 & & & & & & & & & & & 1 \\
\hline Lama sp. & 4 & & & & & & & & & & 1 & 1 \\
\hline Vicugna vicugna & 4 & & & & & & & & & & & 4 \\
\hline Pseudalopex sp. & $2-3$ & & & & & & & & & & & 1 \\
\hline Chaetophractus vellerosus & 2 & & & & & & & & & & 2 & 1 \\
\hline Rodentia & 1 & & 1 & & & & & & 1 & 54 & & 1 \\
\hline Chinchillidae & 2 & & & & & & & & & & & 1 \\
\hline Cricetidae & 1 & & & & & & & & & & & 1 \\
\hline Ctenomys sp. & 1 & & & & & & & & & 9 & & 12 \\
\hline AVES & 1 & & & & & & & & 1 & & & \\
\hline GASTROPODA & 1 & & & & & & & & 3 & 1 & 1 & \\
\hline & Totales & \begin{tabular}{l|l}
4 \\
\end{tabular} & 4 & 1 & 18 & 16 & 93 & 3 & \begin{tabular}{|c|c|}
58 \\
\end{tabular} & 173 & 102 & 242 \\
\hline
\end{tabular}




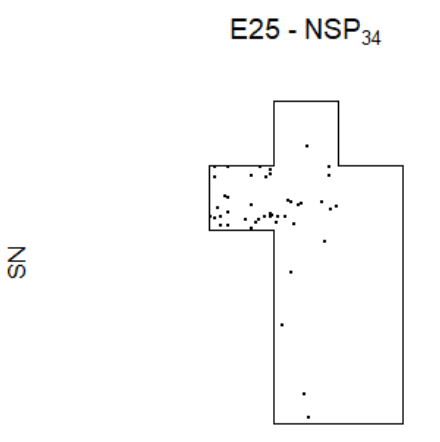

WE

ME
Figura 3. Densidad de especímenes de vertebrados medianos y grandes por cuadrícula de las Estructuras 25 y 93. La densidad está representada por una distribución aleatoria de puntos generada con el paquete MapTools de R.

Figure 3. Density of bone specimens (number of specimens by square, medium to large vertebrates) for structures 25 and 93. Density is represented by a random distribution of points generated under $R$ with the MapTools package. y al suroeste de E93, junto al muro sur, coincidiendo con la mayor concentración de cerámica y lítico. Ambos conjuntos muestran frecuencias similares de termoalteración: 15\% del total de especímenes de la E25 y $5 \%$ del total de la E93.

El 54\% de los restos de vertebrados medianos a grandes de E25 sufrió meteorización (NSP 24, estadios 1-5 de Behrensmeyer), con valores máximos en los estadios 0 (NSP 21) y 3 (NSP 15) de la secuencia. En el caso de E93, la meteorización desciende a $26 \%$ de los vertebrados medianos-grandes (NSP 31, estadios 1-4), con valores máximos en los estadios 0 (NSP 74) y 1 (NSP 13). Una mayor frecuencia relativa de especímenes con meteorización avanzada (estadio 3) sugiere una exposición más prolongada de los materiales de E25.

En ambos conjuntos se registran modificaciones por agentes naturales: carnívoros (E25 NSP 1, E93 NSP 16), roedores (E93 NSP 5) y raíces (E25 NSP 4, E93 NSP 16). No se cuenta con evidencia que permita determinar qué especies carnívoras estaban presentes en Loma l'Ántigo y las marcas podrían atribuirse tanto a animales silvestres del área (e. g. zorros), como a perros domésticos, cuya presencia fue reportada para otros sitios tardíos del Noroeste argentino (Belotti López de Medina, 2016a; Mengoni Goñalons, 1991; Raffino et al., 1977). Los carnívoros pudieron actuar como agentes de destrucción y remoción de fauna (Mondini, 1995; Nasti, 2000), así como de transporte y acumulación a lo largo del poblado.

En cuanto a las trazas antrópicas, el subconjunto de vertebrados medianos-grandes de E25 muestra marcas de corte (NSP 1) y de machacado (NSP 1). E93 registra marcas de corte (NSP 2), machacado (NSP 1), estrías (NSP 2) y hoyos de percusión (NSP 2) y negativos de lascado asociados a fracturas (NSP 6). Estas modificaciones son potenciales indicadores de procesamiento (desarticulación, remoción de carne, fractura). Se destaca la presencia de un artefacto óseo en E93: un fragmento de costilla con una perforación artificial circular (71 mm) (Figura 4). Es posible que se trate de un artefacto similar al tortero de costilla de camélido reportado para el sitio tardío Rincón Chico 15 (Yocavil) (Pratolongo, 2008).

A la presencia de marcas debemos agregar la alta fragmentación de los conjuntos, evidente en los especímenes de huesos largos de vertebrados medianos a grandes (E25 NSP 43, E93 NSP 104). El 75\% de los fragmentos de hueso largo de la E25 mide menos de $30 \mathrm{~mm}$, y menos de $50 \mathrm{~mm}$ en el caso de la E93. La fragmentación probablemente se deba a diferentes causas, ya que se registraron distintas morfologías de borde de fractura; sólo en E93 se registraron 20 fragmentos con bordes de fractura en estado fresco (NSP 20).

Se identificaron 15 especímenes de Camelidae para el piso de E25 y 48 del piso de E93. En E93 se identificaron además Lama sp. (NISP 2) y Vicugna vicugna (NISP 4). La identificación al rango de género o especie se hizo en base a atributos diagnósticos de los incisivos inferiores en el caso de cinco especímenes (dientes sueltos y fragmentos de sínfisis); como exhibían un desgaste considerable, presentamos su asignación taxonómica como tentativa. El espécimen restante de Lama sp. es un calcáneo identificado por morfometría.

Figura 4. Piso de la E93, artefacto sobre costilla. Longitud máxima: $71 \mathrm{~mm}$.

Figure 4. Floor of Structure 93, bone artifact made from a rib. Maximum length: $71 \mathrm{~mm}$.

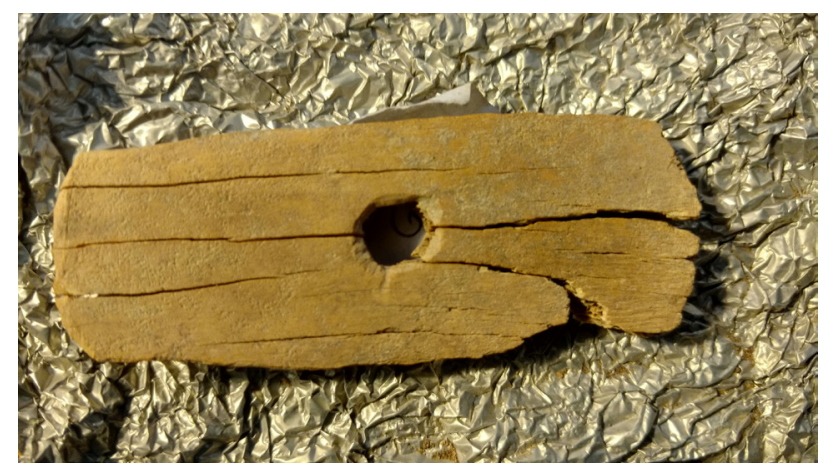


En lo que respecta a los perfiles etarios de Camelidae, en el caso de E25 se identificaron tres especímenes no-epifizados de elementos de fusión intermedia $(<18-$ 36 meses) (De Nigris, 2004); se identificó también una hemimandíbula perteneciente a un animal de entre 9 y 24 meses y un molar superior de un animal mayor a dos años (Wheeler, 1982). Estos datos sugieren la presencia de crías o juveniles y subadultos al menos.

Se determinó la epifización de 10 especímenes de E93, con tres especímenes no fusionados para el grupo de fusión intermedia (<18-36 meses), dos especímenes fusionados para el grupo de fusión temprana (>0-18 meses), cuatro fusionados para intermedia y otro para fusión tardía (>36-48 meses). Se identificaron además dos hemimandíbulas de animales menores a seis meses de edad, una de las cuales conserva incisivos asimilables a Lama sp., y una tercera hemimandíbula con un desgaste correspondiente a los 12-13 años en la secuencia de Wheeler (1982). En resumen, el conjunto de E93 presenta al menos restos de crías-juveniles y de individuos viejos de Camelidae.

Los restos de Camelidae de E25 y E93 (Tabla 3) incluyen elementos apendiculares y axiales, predominando los huesos de la cabeza y de las manos-pies.

\section{Discusión y conclusiones}

Los resultados permiten un primer abordaje zooarqueológico del Período Desarrollos Regionales en el valle del Cajón. Los conjuntos están dominados por Camelidae en el rango familia (ubicuidad y abundancia relativa); se identificó la presencia de especímenes dentro del rango de tamaño de Lama sp., que incluye llama y guanaco, y sínfisis mandibulares que presentan atributos compatibles con Lama sp. y V. vicugna vicugna, si bien

Tabla 3. Número de especímenes identificados de Camelidae por elemento (pisos de ocupación de E25 y E93).

Table 3. Number of identified specimens of the Camelidae family tallied by anatomical element (occupation floors of structures 25 and 93).

\begin{tabular}{|l|r|r|}
\hline \multirow{2}{*}{ Elemento } & E25 & \multicolumn{1}{|c|}{ E93 } \\
\cline { 2 - 3 } Cráneo & 3 & 5 \\
\hline Mandibula & 1 & 10 \\
\hline Diente & 3 & 7 \\
\hline Hioides & 1 & \\
\hline V. cervical & 1 & 1 \\
\hline Costilla & & 1 \\
\hline Escápula & 1 & \\
\hline Húmero & & 3 \\
\hline Radioulna & & 3 \\
\hline Innominado & & 1 \\
\hline Tibia & & 3 \\
\hline Metatarso & & 1 \\
\hline Metapodio & & 8 \\
\hline Astrágalo & & 1 \\
\hline Calcáneo & & 1 \\
\hline H. maleolar & & 1 \\
\hline Falange 2 & & 2 \\
\hline
\end{tabular}

con un desgaste oclusal considerable en el segundo caso. La presencia simultánea de camélidos domésticos y silvestres sería consistente con el registro del sur de los valles Calchaquíes (Belotti López de Medina, 2011, 2016c, 2016b; Izeta, 2007b, 2007a; Pratolongo, 2008) y, en general, con los modelos que postulan una estrategia económica mixta pastoreo-caza (Escola, 2002). Futuras excavaciones y un mayor volumen de especímenes analizados permitirán una descripción más detallada de la estructura taxonómica de los camélidos de Loma l’ántigo.

Se registró la presencia de distintas clases de edad entre los especímenes de Camelidae, pero, por tratarse de muestras muy pequeñas, es difícil establecer tendencias. A lo sumo puede indicarse el promediado de decisiones orientadas a la explotación de animales juvenilessubadultos (palatabilidad de la carne, regulación de rebaño) y de adultos-viejos (sacrificio de animales que agotaron su utilidad como reproductores o como productores de bienes secundarios) dentro de lo que pudo ser un esquema flexible de manejo de rebaños.

Otros taxones que pudieron formar parte de la dieta de las poblaciones de Loma l'Ántigo incluyen grandes roedores (Chinchillidae) y peludos (Chaetophractus vellerosus), pero no se registraron evidencias de procesamiento. Es probable que otros roedores sean intrusivos, ya que su presencia es normal en sitios arqueológicos (e. $g$. (tenomys sp.).

Desde un punto de vista tafonómico regional (sensu Borrero, 1988), destaca la escasez de la fauna recuperada de los pisos de E25 y E93, sobre todo del segundo (NISP 80 al rango de orden o inferior), que fue excavado en su totalidad, en comparación a la mayoría de los recintos del período Formativo del sur de los valles Calchaquíes (e. $g$. NISP 1979 en Cardonal E2, 2161 en Yutopián E11 y 766 en Soria 2 R1) (Belotti López de Medina, 2011; Izeta, 2007b). Esto ya fue observado para recintos de sitios tipo Pukara y otros contextos tardíos del valle de Yocavil (Belotti et al., 2012; Belotti López de Medina, 2013; Pratolongo, 2008). Los mayores conjuntos tardíos provienen de montículos y basurales por fuera de recintos y ubicados en los sectores bajos de los asentamientos (Pratolongo, 2008). Una posible explicación es un cambio en las pautas de mantenimiento de las estructuras residenciales hacia el segundo milenio. La mayor densidad de hallazgos junto a muros y fogones de E93 y E25 puede implicar descartes de facto que escaparon a la limpieza de las habitaciones.

Los arqueotafogramas de la Figura 5 incluyen las excavaciones en área de los pisos de Loma l'Ántigo y de los pisos formativos del sitio Yutopián del valle del Cajón (Izeta, 2007b). Si bien se observan diferencias entre pares de conjuntos, los valores de Loma l'Ántigo se encuentran dentro del rango de las medidas de los conjuntos de Yutopián. Esto sugiere que las historias tafonómicas de los conjuntos, que incluyen procesos artificiales y naturales, 


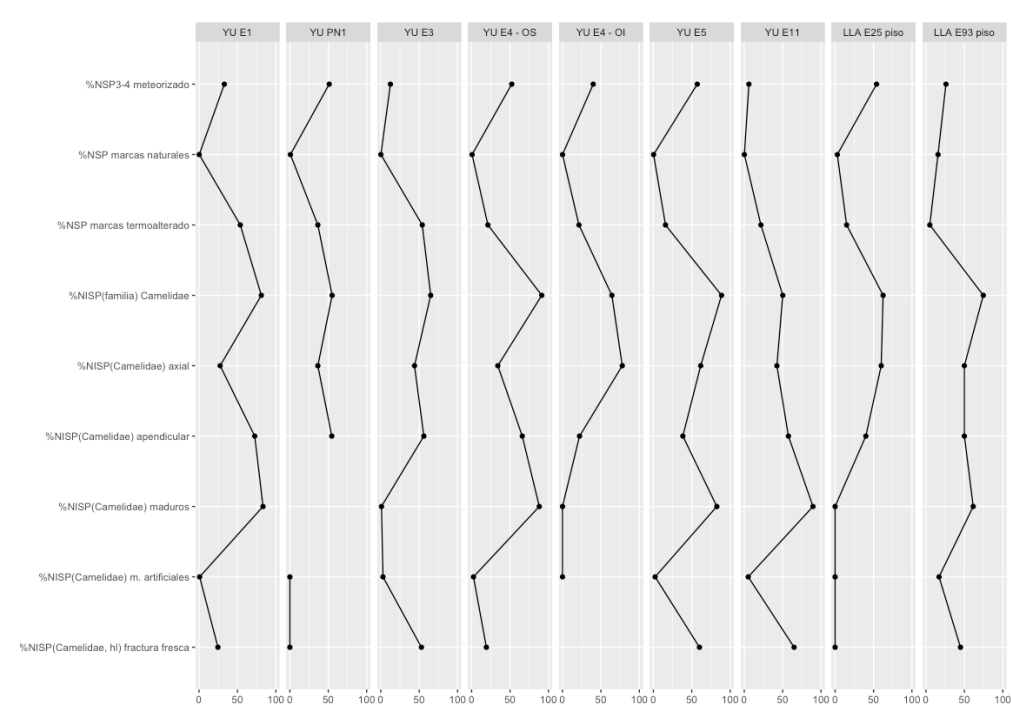

Figura 5. Arqueotafogramas de Yutopián (Estructuras 1, 3, 4 ocupaciones inferior y superior, 5, 11 y Patio Núcleo 1) y pisos de ocupación de Loma l'Ántigo E25 y E93.

Figure 5. Archaeotaphograms for Yutopián (Structures 1, 3, 4 -inferior and superior occupations-, 5, 11, Core Courtyard 1) and occupation floors from Loma l'Ántigo (structures 25 and 93). estuvieron sujetas a similares restricciones ambientales y socioculturales durante ambos períodos. Esto aplica en primer lugar a procesos postdepositacionales destructivos y de remoción, como modificación por carnívoros, meteorización o la termoalteración post-descarte. Las variaciones entre pares de conjuntos individuales, del mismo período o no, pueden implicar condiciones particulares, como diferencias funcionales entre contextos de acumulación. Este problema deberá ser abordado en futuros trabajos.

La diversidad taxonómica de Loma l'Ántigo es similar a la de los conjuntos de Yutopián y la Estructura 2 de Cardonal. El rango de familias registradas puede atribuirse a la continuidad espacial y temporal de prácticas tecnoeconómicas y factores medioambientales, que afectan tanto al consumo humano como a la presencia de especies comensales y perturbadoras en el sitio. Camelidae es la familia predominante en todos los conjuntos. Izeta (2007a, 2007b) identificó la presencia de especímenes compatibles con los morfotipos de vicuña, llama y guanaco en Cardonal y Yutopián, algo observado también en sitios de Yocavil. La información taxonómica de Loma l'Ántigo es todavía insuficiente, pero consistente con este patrón.

Con independencia del período, al comparar Cardonal, Yutopián y Loma l'Ántigo se observa una notable variación para los perfiles de supervivencia/mortandad, a juzgar por la frecuencia relativa de fragmentos osteológicamente maduros y series dentales completas de Camelidae. Lo mismo parece ocurrir con la relación entre fragmentos apendiculares y axiales de Camelidae, si bien el esqueleto apendicular tiende a ser predominante.

En un contexto regional más amplio, el predominio de Camelidae y la presencia de especies silvestres y domésticas son pautas comunes a los sitios agroalfareros de los valles Calchaquíes y otras cuencas de la región valliserrana (e. g. Belotti López de Medina, 2015b, 2015a;
Belotti López de Medina et al., 2016; de Guerrero, 1968; Izeta, 2007b; Mengoni Goñalons, 1991, 2013; Mercolli, 2011; Pratolongo, 2008), así como de la Puna (Grant, 2010; Izeta, 2008; Olivera, 1997; Yacobaccio et al., 1997). En cuanto a otras tendencias postuladas en trabajos previos, Loma l'Ántigo no muestra una mayor frecuencia de camélidos domésticos en comparación con los sitios tempranos del valle. Respecto a los perfiles de edad de Camelidae, E25 presenta restos de animales menores a tres años únicamente, mientras que E93 muestra una supervivencia del NISP\% 33 (dentición) al NISP\% 57 (epifización) para los primeros 0-36 meses. Estos valores implican una menor frecuencia de animales maduros en comparación con al menos cuatro de los conjuntos de Yutopián; por lo tanto, tampoco puede hablarse todavía de una tendencia diacrónica en cuanto a los perfiles etarios de los conjuntos.

A modo de conclusión y de hipótesis de trabajo para contrastar con futuras evidencias, proponemos que el registro zooarqueológico de Loma l'Ántigo continúa las tendencias observadas en Cardonal y Yutopián para el período Formativo. Como corolario, en principio no se observan cambios que puedan vincularse a los procesos de jerarquización y centralización del segundo milenio. Esta continuidad sugiere más bien una tafonomía regional determinada por un conjunto estable de factores medioambientales y sociales, que incluye a las prácticas tecnoeconómicas de los habitantes de Loma l'Ántigo. No obstante, los conjuntos de Loma l'Ántigo son pequeños y esto implica que medidas como la riqueza taxonómica estén sujetas a errores de muestreo.

Las variaciones coetáneas intra e inter-sitio de los conjuntos pueden atribuirse, por el momento, a la acumulación de decisiones económicas y procesos tafonómicos contingentes dentro de un único complejo tecnológico y ambiental regional relativamente constante. Esto no excluye cambios a lo largo de 1500 años, menos tratándose de una estrategia económica caracterizada 
como flexible y adversa al riesgo, pero la observación de cambios regionales de larga duración requerirá de la agregación de datos de numerosos conjuntos y sitios del valle, tarea colectiva que está en curso.

\section{Agradecimientos}

Esta investigación se llevó a cabo en el marco del proyecto PICT 2011 Raíces 636 (ANPCyT). En los trabajos de campo participaron Leda Moro, Sofía Fernández Sancha y los miembros del equipo PasCal. También agradecemos a los evaluadores y a los editores de la Revista del Museo de Antropología, cuyas críticas y sugerencias contribuyeron al presente trabajo.

Nuestro agradecimiento especial a la familia Pachao de Ovejería Chica por su hospitalidad y apoyo en nuestro trabajo. A la municipalidad de San José por su colaboración en la logística.

Para la generación de las cartas temáticas se utilizaron modelos de elevación digital y vectores del Instituto Geográfico Nacional de la República Argentina.

\section{Referencias}

Behrensmeyer, A. K. (1978). Taphonomic and ecologic information from bone weathering. Paleobiology, 1, 150-162.

Belotti, C., Gaal, E., Greco, C., \& Raffaele, L. (2012). Volviendo a la Loma Rica de Shiquimil. Informe sobre el trabajo de campo de año 2009. En Entre Pasados y Presentes 3. Estudios contemporáneos en Ciencias Antropologicas (pp. 910-923). Mnemosyne.

Belotti López de Medina, C. R. (2011). Zooarqueología del sitio formativo Soria 2, valle de Yocavil (Catamarca), siglo I d.C. Revista del Museo de Antropología, 4, 3-16.

Belotti López de Medina, C. R. (2013). Usos económicos y rituales de la fauna en la región valliserrana del Noroeste argentino entre los inicios del periodo Temprano y hasta la conquista Inka (ca. 600 AC - 1600 DC): Zooarqueología del valle de Yocavil (Catamarca), centro y norte del Valle Calchaquí (Salta) y la Quebrada de Humahuaca (Jujuy). Universidad de Buenos Aires.

Belotti López de Medina, C. R. (2015a). Desigualdad e intensificación de la subsistencia en el valle de Yocavil (Catamarca y Tucumán, Argentina) entre los siglos I a.C y XVI d.C. Relaciones de la Sociedad Argentina de Antropología, 40(1), 73-100.

Belotti López de Medina, C. R. (2015b). Subsistence and Economy at the Calchaquí Valley (Salta, Argentina) during the Regional Developments Period (ca. 1000-1430 AD): Zooarchaeology of Las Pailas locality. Journal of Archaeological Science: Reports, 4, 461-476. https://doi. org/10.1016/j.jasrep.2015.10.016

Belotti López de Medina, C. R. (2016a). Morphometry of a Pre-Hispanic Dog from Northwestern Argentina (AD 800-1200). International Journal of Osteoarchaeology, n/a-n/a. https://doi.org/10.1002/oa.2577

Belotti López de Medina, C. R. (2016b). Nuevos datos zooarqueológicos de Rincón Chico 15, Valle de Yocavil (Catamarca, Argentina). Arqueología, 23, en prensa-en prensa.

Belotti López de Medina, C. R. (2016c). Zooarqueología de un centro administrativo inca del Valle de Yocavil (Catamarca): El Tambo de Punta de Balasto. Revista del Museo de Antropología, 9(2), 17-27.

Belotti López de Medina, C. R., López Geronazzo, L., \& Otero, C. (2016). At the Feet of the Fortress: Analysis of Inka Period (ca. AD 1430-1536) Archaeofaunal Assemblages from Residential Unit 1 (RU1), Pucara de Tilcara (Jujuy, Argentina). PLOS ONE, 11(10), e0163766-e0163766. https://doi.org/10.1371/journal.pone.0163766

Borrero, L. A. (1988). Tafonomía regional. En N. Ratto \& A. Háber (Eds.), De contextos, procesos y otros huesos. ICA (FFyL-UBA).

Bugliani, M. F. (2012). Loma L'Ántigo: Consideraciones sobre la arquitectura de un poblado tardío en el valle del Cajón (Catamarca, Argentina). Comechingonia, 16, 71-84.

Bugliani, M. F. (2018). Evidencias del Tardío en el Valle del Cajón (Catamarca). Una mirada desde el sitio Loma I'Ántigo. Relaciones de la Sociedad Argentina de Antropología, 43(1), 35-54.

Bunn, H. T. (1981). Archaeological evidence for meateating by Plio-Pleistocene hominids from Koobi Fora and Olduvai Gorge. Nature, 291(18), 574-577.

Burger, R. L., \& Salazar, L. C. (2003). The 1912 Yale Peruvian Scientific Expedition Collections from Machu Picchu: Human and Animal Remains. Yale University Press. http://books.google.com.ar/books?id=iS6zbwAACAAJ

Carlson, D. L. (2017). Quantitative Methods in Archaeology Using R. Cambridge University Press.

de Guerrero, L. R. (1968). Análisis: Restos óseos de animales-SSalLap2.

De Nigris, M. (2004). El consumo en grupos cazadores recolectores. Un ejemplo zooarqueológico de Patagonia meridional. Sociedad Argentina de Antropología.

Escola, P. S. (2002). Caza y pastoralismo: Un reaseguro 
para la subsistencia. Relaciones de la Sociedad Argentina de Antropología, 27, 233-245.

Fernandez-Jalvo, Y., \& Andrews, P. (2016). Atlas of taphonomic identifications: 1001+ images of fossil and recent mammal bone modification. Springer.

Fisher, J. W. (1995). Bone surface modifications in zooarchaeology. Journal of Archaeological Method and Theory, 2(1), 7-68. https://doi.org/10.1007/bf02228434

González, L. R., \& Tarragó, M. N. (2005). Vientos del sur. El valle de Yocavil (Noroeste Argentino) bajo la dominación incaica. Estudios Atacameños, 29, 67-95.

Grant, J. (2010). Aportes de distintas técnicas osteométricas para la identificación interespecífica de camélidos sudamericanos. En M. A. Gutiérrez, M. De Nigris, P. M. Fernández, M. Giardina, A. Gil, A. D. Izeta, G. Neme, \& H. D. Yacobaccio (Eds.), Zooarqueología a principios del siglo XXI. Aportes teóricos, metodológicos y casos de estudio (pp. 17-28). Ediciones del Espinillo.

Hillson, S. (2005). Teeth. Cambridge University Press.

Izeta, A. D. (2007a). Zooarqueología del Formativo del valle del Cajón (Catamarca, Argentina). En Pacarina (Vol. 1, pp. 471-477). Número especial de la revista Pacarina, FHyCS-UNJu.

Izeta, A. D. (2007b). Zooarqueología del sur de los valles Calchaquíes (Provincias de Catamarca y Tucumán, República Argentina). British Archaeological Reports.

Izeta, A. D. (2008). Late Holocene camelid use tendencies in two different ecological zones of Northwestern Argentina. Quaternary International, 180, 135-144. https://doi.org/10.1016/j.quaint.2007.08.026

Izeta, A. D., \& Cortés, L. I. (2006). South American camelid palaeopathologies: Examples from Loma Alta (Catamarca, Argentina). International Journal of Osteoarchaeology, 16(3), 269-275. https://doi.org/10.1002/oa.823

Izeta, A. D., Srur, M. G., \& Labarca, R. (2012). Guía de osteometría de camélidos sudaméricanos. Universidad Nacional de Córdoba.

Izeta, A. D., Urquiza, S. V., \& Baldini, L. (2009). La arqueofauna del período Tardío en el NOA. Una aproximación desde los conjuntos del sitio Molinos I (provincia de Salta, R. Argentina). Arqueología, 15, 63-84.

Johnson, E. (1985). Current developments in bone technology. En M. B. Schiffer (Ed.), Advances in Archaeological Method and Theory (Vol. 8, pp. 157-235). Academic Press.
Kaufmann, C. A., Álvarez, M. C., \& Pérez, S. I. (2017). Estimación de la edad de guanaco (Lama guanicoe) a partir de los dientes incisivos. Revista del Museo de Antropología, 10.

Kent, J. D. (1982). The Domestication and Exploitation of the South American camelids: Methods of analysis and their application to circum-lacustrine archaeological sites in Bolivia and Perú.

Lyman, R. L. (1994). Vertebrate Taphonomy. Cambridge University Press.

Lyman, R. L. (2008). Quantitative paleozoology (14996216). Cambridge University Press. http://www. loc.gov/catdir/enhancements/fy0803/2007036203-b. html http://www.loc.gov/catdir/enhancements/ fy0803/2007036203-t.html http://www.loc.gov/catdir/ enhancements/fy0803/2007036203-d.html

Menegaz, A., Salemme, M., \& Ortiz Jaureguizar, E. (1988). Una propuesta de sistematización de caracteres morfométricos de los metapodios y las falanges de Camelidae. En N. Ratto \& A. Haber (Eds.), De procesos, contextos y otros huesos (pp. 53-64). FFyL-ICA.

Mengoni Goñalons, G. L. (1991). Análisis de los restos óseos de camélido recuperados en la localidad de "Las Pailas» (Salta).

Mengoni Goñalons, G. L. (1999). Cazadores de guanacos de la estepa patagónica. Sociedad Argentina de Antropología.

Mengoni Goñalons, G. L. (2013). El aprovechamiento de fauna en sociedades complejas: Aspectos metodológicos y su aplicación en diferentes contextos arqueológicos del NOA. En V. I. Williams \& M. B. Cremonte (Eds.), Al borde del imperio, paisajes sociales, materialidad y memoria en áreas periféricas del noroeste argentino (pp. 311-396). Publicaciones de la Sociedad Argentina de Antropología.

Mercolli, P. H. (2011). El truco está en la diversidad: Estrategias de manejo pastoril en la Quebrada de Humahuaca, provincia de Jujuy, Argentina, entre el 900 y el 1.200 AD. Arqueología, 17, 81-99.

Mondini, N. M. (1995). Artiodactyl Prey Transport by Foxes in Puna Rock Shelters. Current Anthropology, 36(3), 520524. https://doi.org/10.1086/204391

Morlan, R. E. (1983). Spiral Fractures on Limb Bones: Which ones are artificial? En G. M. LeMaine \& S. McEachern (Eds.), Carnivores, Human Scavengers and Predators: A Question of Bone Technology, Proceedings of the Fifteenth Annual Conference (pp. 241-269). The Archaeological Association of the University of Calgary. 
Moro, L. (2016). Análisis del material lítico del sitio Loma l'Ántigo. En Actas del XIX Congreso Nacional de Arqueología Argentina (pp. 135-138).

Nasti, A. (2000). Modification of Vicuña Carcasses in HighAltitude Deserts. Current Anthropology, 41(2), 279-283. https://doi.org/10.1086/300133

Nastri, J., Coll Moritán, V., Belotti López de Medina, C. R., Stern Gelman, L., \& Schaeffers, F. (2012). El Intermedio Tardío en la Sierra del Cajón (provincia de Catamarca). Avance de las investigaciones en Morro del Fraile. Estudios Sociales del NOA-Nueva Serie, 12, 81-110.

Olivera, D. E. (1997). La importancia del recurso Camelidae en la Puna de Atacama entre los 10.000 y 500 años A.P. Estudios Atacameños, 14, 29-41.

Pacheco Torres, V. R., Altamirano Enciso, A., \& Guerra Porras, E. S. (1979). Guia osteológica de camélidos sudamericanos. Universidad Nacional Mayor de San Marcos.

Potts, R., \& Shipman, P. (1981). Cutmarks made by stone tools on bones from Olduvai Gorge, Tanzania. Nature, 291(18), 577-580.

Pratolongo, G. J. (2008). Estudio de los restos faunísticos de dos sitios tardíos en el valle de Yocavil, provincia de Catamarca: Rincón Chico 15 y Las Mojarras 1. En M. N. Tarragó \& L. R. González (Eds.), Estudios arqueológicos en Yocavil (pp. 81-126). Asociación de Amigos del Museo Etnográfico.

Raffino, R., Tonni, E., \& Cione, A. L. (1977). Recursos alimentarios y economía en la Región de la Quebrada del Toro, Provincia de Salta, Argentina. Relaciones de la Sociedad Argentina de Antropología, 11 NS, 9-30.

Rodriguez Loredo, C. (1992). Étude archéozoologique du site inca Potrero-Chaquiago: Quartier Retambay, Andalgalá, Pcia. De Catamarca, Argentine [Doctoral]. Pantéon-Sorbonne.

Salemme, M., Miotti, L., \& Tonni, E. (1988). La determinación sistemática de los mamíferos en el análisis arqueofaunístico. En A. F. Haber \& N. Ratto (Eds.), De procesos, contextos y otros huesos (pp. 65-73).
Facultad de Filosofía y Letras (UBA), Instituto de Ciencias Antropológicas.

Scattolin, M. C. (2006). De las comunidades aldeanas a los curacazgos en el Noroeste argentino. Boletín de arqueología PUCP, 10, 357-398.

Scattolin, M. C., \& Gero, J. M. (1997). Consideraciones sobre fechados radiocarbónicos de Yutopián (Catamarca, Argentina). 3, 352-357.

Scattolin, M. C., Pereyra Domingorena, L., Cortés, L. I., Bugliani, M. F., Calo, C. M., Izeta, A. D., \& Lazzari, M. (2007). Cardonal: Una aldea formativa entre los territorios de valles y puna. Cuadernos de la Facultad de Humanidades y Ciencias Sociales, 32, 211-225.

Tarragó, M. N. (2000). Chacras y Pukara. Desarrollos sociales tardíos. En M. N. Tarragó (Ed.), Los Pueblos Originarios y la Conquista (Vol. 1, pp. 257-300). Editorial Sudamericana.

Ten Kate, H. F. (1893). Rapport sommaire sur une excursion archéologique dans les provinces de Catamarca, de Tucumán et Salta. Revista del Museo de La Plata, 5, 331-384.

Turner, J. C. M. (1973). Descripción geológica. Laguna Blanca, hoja 11d Escala: 1: 200.000. Ministerio de Economía de la Nación, Secretaría de Industria y Minería.

Weiser, W. (1922). Diario de la 4ta Expedición Benjamin Muniz Barreto. Departamento Arqueología. Museo de La Plata.

Wheeler, J. C. (1982). Aging llamas and alpacas by their teeth. Llama World, 1(2), 12-17.

Wolverton, S., Dombrosky, J., \& Lyman, R. L. (2014). Practical Significance: Ordinal Scale Data and Effect Size in Zooarchaeology. International Journal of Osteoarchaeology, 26(2), 255-265. https://doi. org/10.1002/oa.2416

Yacobaccio, H. D., Madero, C. M., Malmierca, M. P., \& Reigadas, M. del C. (1997). Caza, domesticación y pastoreo de camélidos en la Puna argentina. Relaciones de la Sociedad Argentina de Antropología, 22-23, 389-418. 